\title{
Massive Intracerebral Haemorrage Escaped Detection by Cerebral Oximetry
}

\author{
Skhirtladze-Dworschak K, Felli A, Opfermann P, Dworschak M \\ Medical University of Vienna, Dept. of Anaesthesia, Critical Care \& Pain Medicine \\ Waehringer Guertel 18-20, Vienna, Austria
}

Background: Cerebral oximetry employing near infrared spectroscopy (NIRS) is a non-invasive brain monitor. It is predominantly used to detect imminent alterations in the cerebral circulation that require urgent action to avoid major injury.

Case report: A 50 year old female patient was admitted to our intensive care unit after heart transplantation because of arrhythmogenic cardiomyopathy. The initial course was uneventful; she could be extubated on the following day and required only moderate inotropic support. Five days later, however, extracorporeal membrane oxygenation (ECMO) had to be instituted following CPR because of low cardiac output due to acute transplant rejection. At the same time, bilateral NIRS monitoring was begun, which depicted \% values in the low to high 40 s with constantly lower $(-8 \%)$ saturations over the right forehead. Anisocoria (right > left) was first noted after two days on ECMO. Subsequently, the right pupil did not show a positive light reflex anymore. NIRS values as well as haemodynamics were stable throughout all these neurological changes. A CT-scan of the brain revealed a massive intracerebral bleeding within the right temporal lobe that necessitated evacuation and osteoclastic craniotomy because of accompanying brain oedema after which pupils became isocoric again.

Discussion: Cerebral oximetry obviously missed detection of this live-threatening brain haemorrhage in the supply zone of the middle cerebral artery. The observed phenomenon can either be explained by the distance between the ipsilateral optode and the bleeding or by the fact that NIRS is not able to distinguish between haemoglobin-bound oxygen that is located within the vasculature and that outside of blood vessels, which in this case had accumulated in the parenchymal tissue of the temporal lobe. Furthermore, intracerebral haematomas absorb light less intensely as extra cerebral ones hampering their identification (1).

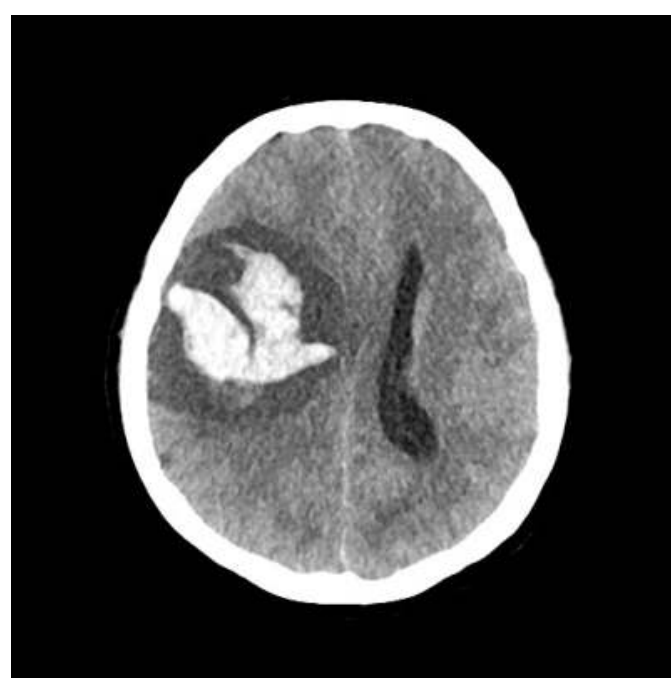

Image 1) CCT prior to craniotomy

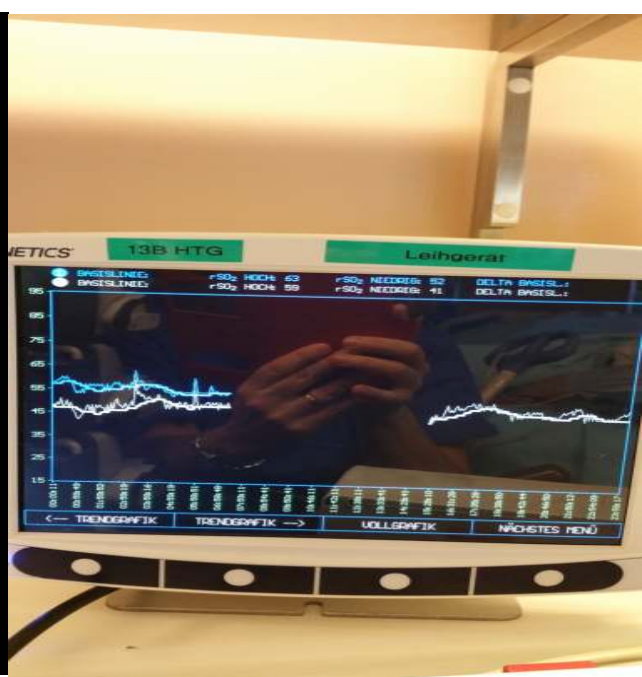

Image 2) INVOS $5100^{\circledR}$ readings Sensor 1 (Blue) Right hemisphere Sensor 2 (White) Left hemisphere

Learning points and Conclusion: Severe brain damage located primarily in the supply zone of the middle cerebral artery may not be detected by monitoring cerebral tissue oxygenation with the help of near infrared spectroscopy when optodes are placed on the forehead.

\section{References:}

1) Gopinath SP et al. J Neurosurg $83 ; 438-44,1995$ 